\title{
Current treatment strategies in pediatric gastrointestinal stromal cell tumor
}

\author{
Brent A. Willobee, Hallie J. Quiroz, Matthew S. Sussman, Chad M. Thorson, Juan E. Sola, Eduardo A. Perez \\ Department of Surgery, Miller School of Medicine, University of Miami, Coral Gables, FL, USA \\ Contributions: (I) Conception and design: All authors; (II) Administrative support: All authors; (III) Provision of study materials or patients: All \\ authors; (IV) Collection and assembly of data: All authors; (V) Data analysis and interpretation: All authors; (VI) Manuscript writing: All authors; (VII) \\ Final approval of manuscript: All authors. \\ Correspondence to: Eduardo A. Perez. Department of Surgery, Miller School of Medicine, University of Miami, Coral Gables, FL, USA. \\ Email: eperez3@med.miami.edu.
}

\begin{abstract}
Gastrointestinal stromal tumors (GIST) are exceedingly rare tumors in the pediatric population. As a result, many clinicians either may never see this diagnosis or will encounter it only a few times throughout their careers. Additionally, the more we discover about this disease, it becomes evident that it represents a distinct clinical entity from adult GIST. Many of the treatments and strategies used to combat the adult tumor are either ineffective or may be harmful to the pediatric population with this disease. The unique tumor biology found in pediatric GIST necessitates unique approaches and treatment strategies in order to achieve the best clinical outcome. This review aims to discuss the most recent data available on the different therapeutic modalities utilized in cases of Pediatric GIST.
\end{abstract}

Keywords: Pediatric; wild-type (WT); gastrointestinal stromal tumor (GIST); review; treatment

Received: 26 June 2018; Accepted: 23 July 2018; Published: 08 August 2018.

doi: $10.21037 / \operatorname{tgh} .2018 .07 .09$

View this article at: http://dx.doi.org/10.21037/tgh.2018.07.09

\section{Introduction}

Gastrointestinal stromal cell tumors (GIST) are sarcomatous tumors which arise from the pacemaker cells of the GI tract known as the interstitial cells of Cajal. Although GIST are the most common sarcoma of the GI tract, they remain quite rare. In the United States, recent analysis of the National Cancer Institute's (NCI) Surveillance, Epidemiology, and End Results (SEER) data suggests the total incidence of all GIST is 6.7 cases per million subjects (1). Recent global estimates vary broadly depending upon the population studied, but generally range from 5 to 19 cases per million subjects (2,3). GIST in pediatric patients are even more rare. Their exact incidence is difficult to classify due to rarity and evolving definitions about what constitutes a case of pediatric GIST. However, SEER data estimates the incidence of GIST in subjects aged between 8-20 years old to be approximately 0.11 cases per million subjects or $1.64 \%$ of all GIST cases (1). In addition to being quite rare, the existing literature on pediatric GIST demonstrates key differences in clinical presentations, tumor biology, and patient outcomes when compared to adult cases (4-6). Due to the rarity and the unique features of pediatric GIST, the National Comprehensive Cancer Network (NCCN) task force recommends referral to specialty treatment centers if there is a suspicion or diagnosis of pediatric GIST (7). The remainder of this article will focus on the multimodal approaches to the treatment of these rare, indolent pediatric tumors.

\section{Surgery}

Even with the advent of molecularly targeted therapies, complete surgical resection, while avoiding pseudocapsule rupture, currently remains the only curative treatment modality for GIST (7-10). For oncologic resections performed with curative intent, the commonly accepted goal of therapy is to achieve histologically negative margins 
on the resected specimen. This has been shown to impart an event free survival (EFS) benefit in some cancers but its absolute importance in the treatment of GIST remains unclear. Some adult studies seem to support the conclusion that an $\mathrm{R} 0$ resection, or histologically negative margins, imparts an increase in EFS $(11,12)$. However, numerous other studies fail to find a statistically significant difference in EFS between an R0 and an R1 resection $(13,14)$. It is likely that tumor biology, size, and location are more predictive of tumor recurrence and long-term survival than $\mathrm{R} 0$ vs. R1 resection status.

This paradigm is further supported in studies of pediatric or wild type (WT) GIST. In a study of 76 patients with pediatric GIST, Weldon et al. demonstrated disease progression rate of $84 \%$ over 10 years postoperatively despite having comparable rates of $\mathrm{R} 0$ resection to those in adult series (15). The only significant predictors of disease progression were tumor mitotic rate and metastasis at presentation. These recurrences are less likely to be local or regional and are found at distant sites in $71 \%$ of cases (15). Additionally, pathologic studies of the growth pattern of pediatric/WT GIST reveal a multifocal, nodular growth pattern. On final pathology, histologically normal tissue is frequently found separating islands of tumor cells, calling into question the veracity of even those cases with supposed "negative" margins (16). In spite of this seemingly aggressive nature, the complete natural history of Pediatric/WT GIST reveals a more indolent disease than the adult counterpart. Although findings must be interpreted in light of the small numbers involved in these case series, two separate studies have shown 10-year survival rates between $92-94 \%$ despite disease recurrence rates between $70-80 \%(8,15)$.

Taking into account the literature reporting equivocal outcomes of R0 vs. R1 resections for Pediatric/WT GIST, the high likelihood of disease progression, and the relatively indolent nature of the tumor, it is recommended that an organ sparing approach be adopted whenever possible. While an R0 resection is good oncologic practice and attempted whenever feasible, the surgeon must always take into account the immediate risk to the patient and long-term morbidity of the surgery in light of the dubious clinical benefit in these cases. This strategy of organ sparing and wedge resections has support in multiple case series $(8,15)$.

The initial surgery for Pediatric/WT GIST bears some key differences from the surgery for the adult tumor. The use of frozen sections is encouraged to aid in achieving a negative margin status. However, this recommendation should be interpreted in light of the fact that re-excisions in an effort to achieve negative microscopic margins may not be justified if they result in significantly increased morbidity. In addition to resection of the primary lesion, the entire peritoneum and retroperitoneum should be inspected for occult lesions which may be biopsied for staging purposes. Complete resection of any distant tumor deposits should only be undertaken if an occult lesion presents a clear future risk for causing obstruction, hemorrhage, or perforation. Tumor spread to lymph node basins is much more common in pediatric GIST than in the adult counterpart. Therefore, while lymph node sampling is not a common part of surgery for adult GIST, all lymph node basins draining the primary tumor should be explored and any suspicious appearing nodes should be removed. Additionally, if signs of lymphatic spread have been identified preoperatively, these nodes should be sampled during the surgery to confirm metastatic spread. As it is unlikely to increase EFS, a complete or formal lymphadenectomy of these basins is not supported by current evidence given the additional risk for morbidity from these procedures. Finally, the liver should be explored visually or by preoperative and/or intraoperative ultrasound to identify any suspicious lesions for biopsy when technically feasible. A formal liver resection should not be attempted as it is unlikely to impart any additional benefit to the patient (9).

The technique used in the initial surgery is left largely up to the primary surgeon. Tumors larger than $10 \mathrm{~cm}$ are more likely to benefit from an open procedure, but laparoscopic techniques are suitable when their use does not increase the risk for rupture of the pseudocapsule or morbidity. In general, the surgeon should utilize the technique with which they are most familiar and best trained. While the use of primary endoscopic resections is not routinely recommended, it has been described for small, superficial tumors. Joint laparoendoscopic techniques for gastric GIST have also recently been described in adults and preliminary data from their use is highly encouraging. A key advantage to these techniques is the reduction in the total volume of gastric wall resected while still achieving a complete, $\mathrm{R} 0$ resection margin $(17,18)$. This is clearly advantageous in pediatric GIST as one of the primary goals of surgery is to minimize both long and short-term morbidity. However, these procedures require a surgeon skilled in both laparoscopic and endoscopic techniques and will likely require further validation before their use will achieve wide 


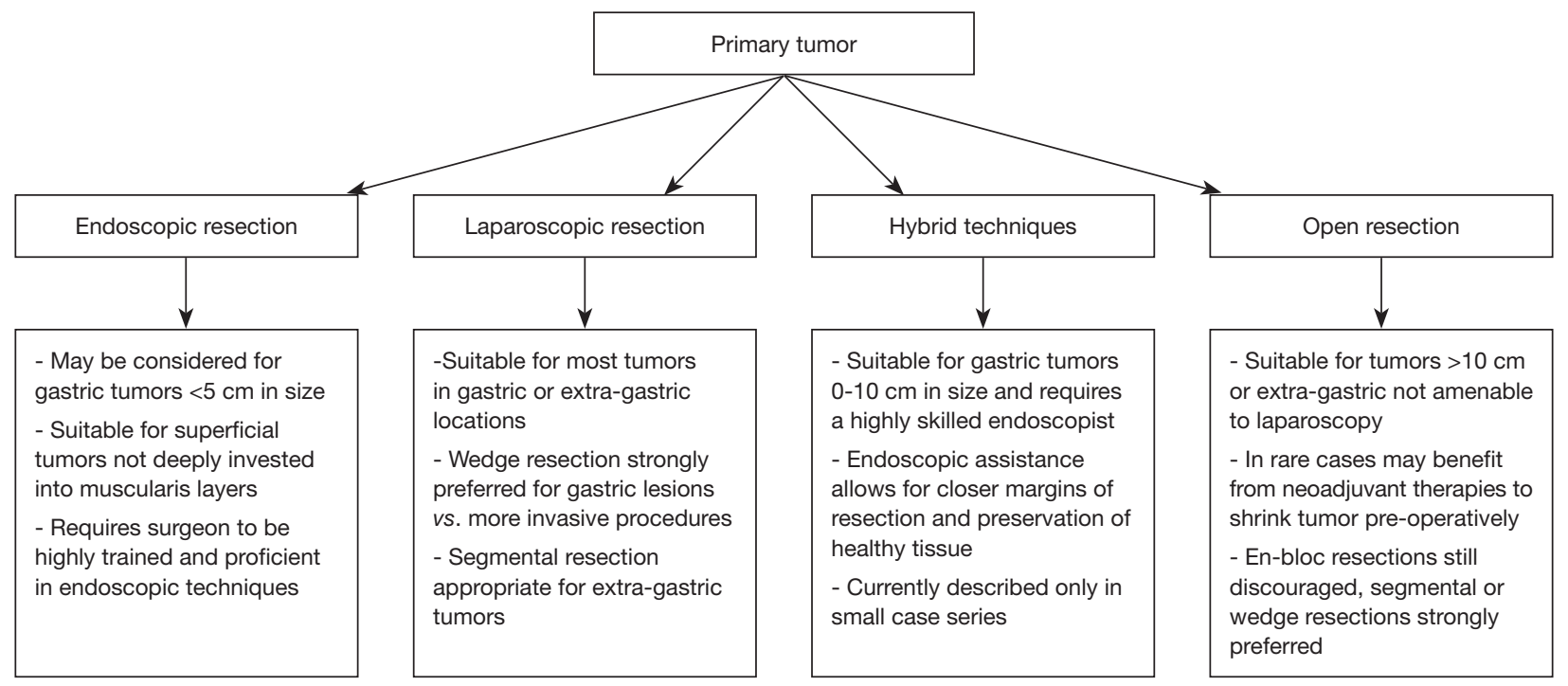

Figure 1 Surgical treatment algorithm for primary tumors.

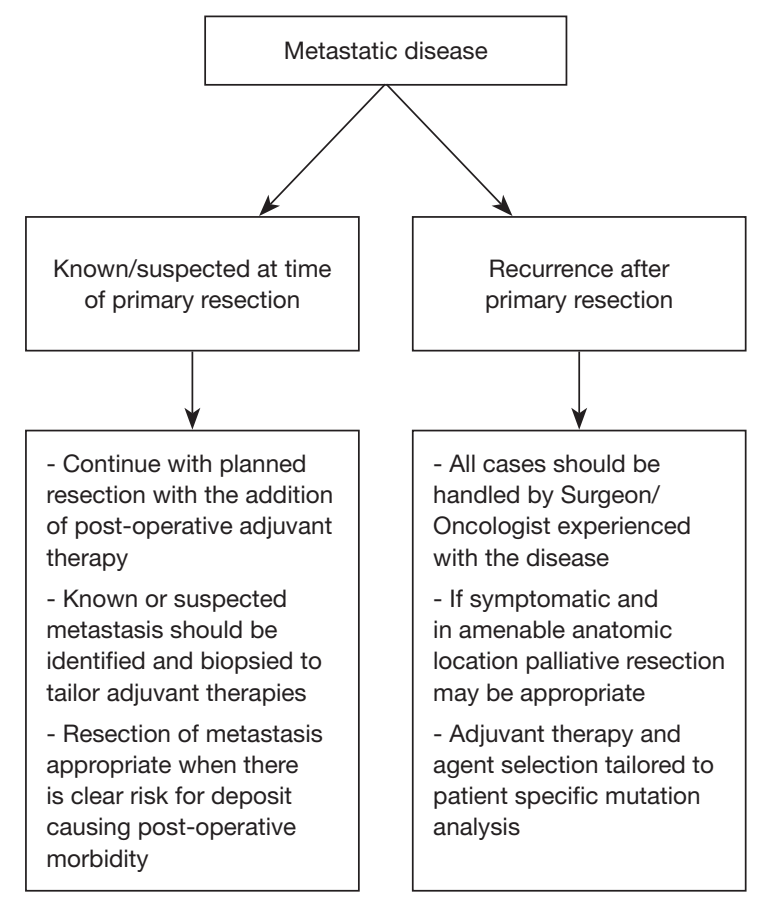

Figure 2 Surgical treatment algorithm for metastatic disease.

acceptance. A summary of the various surgical treatment modalities available for treatment of the primary tumor can be found in Figure 1.

The overall theme of these recommendations can be simplified to say that improving the long term functional status of the patient takes priority over an aggressive, "no tumor left behind" approach during the initial surgery. The role for resection of metastatic deposits is only for diagnosis and staging at the time of the initial operation as well as for those likely to cause significant symptoms in the near future. Extensive metastasectomy is not indicated as it increases morbidity and does not result in any proven clinical benefit. However, a key point to be made is that the presence of metastatic disease should not preclude resection of the primary tumor as studies have shown that surgery, even in the presence of metastatic disease, offers a clear survival benefit in these patients (19). Finally, the role of surgery for recurrent disease, whether local or metastatic, remains unclear and is in need of further study. However, some studies have already found that repeat resections for pediatric GIST have a statistically significant, independent association with decreased EFS (15). These findings will require further research as they are subject to numerous confounding variables. A summary of these principles in the treatment of metastatic disease can be found in Figure 2.

\section{Adjuvant therapies}

The treatment of adult GIST was revolutionized in 1998 by the discovery that activating KIT mutations were a primary feature in the biology and progression of these tumors (20). Prior to this, there were no diagnostic codes specifically identifying GIST as a unique subtype of sarcoma and they were often lumped in with more aggressive subtypes of intestinal sarcoma $(1,3)$. However, their identification as a 
unique type of tumor with a unique biology occurred nearly simultaneously with the arrival of tyrosine kinase inhibitors (TKIs) which were being developed for the treatment of chronic myelogenous leukemia. This ushered in a new era in the treatment of GIST. TKIs are now the mainstay of treatment for metastatic adult GIST and for certain highrisk subtypes of localized disease (21). In rare circumstances, they are even utilized in a neoadjuvant fashion for advanced, localized disease when tumor shrinkage would allow for decreased perioperative morbidity $(22,23)$. However, this last strategy is even more rarely used in the pediatric population as certain case reports have suggested worse outcomes when using this approach (24).

Unfortunately, the majority of Pediatric/WT GIST do not express these mutations in KIT and PDGFRA, and consequently have proven less responsive to traditional TKI treatment. The predominant mutation in Pediatric/WT GIST is an inactivating mutation in the SDH gene complex. Despite this being the most common mutation, these tumors are highly heterogeneous as a group and are associated with numerous other activating mutations primarily in FGF4 (fibroblast growth factor 4), BAALC (brain and acute leukemia, cytoplasmic), IGF1R (insulin-like growth factor I receptor), NELL1 (NEL-like 1), CRLF1 (cytokine receptor-like factor 1), PLAG1 (pleomorphic adenoma gene 1), and $F G F 3$ (fibroblast growth factor 3) (16). Interestingly, tumor biology also seems to relate to the anatomy in WT and pediatric GIST as SDH-deficient tumors appear to be exclusively gastric in location (25). Due to the complexity of the mutations involved in pediatric/WT GIST and its consequences on therapeutic management, it cannot be stressed enough the importance of referral to specialized centers when determining adjuvant therapies for the pediatric patient presenting with GIST.

Similar to the treatment of adult GIST, if the primary tumor is completely resected at the time of the initial surgery with no tumor spillage, then no adjuvant therapy is warranted. However, the patient will need close followup given the high rate of recurrence in these cases. In the case of non-localized tumor, adjuvant therapy with imatinib has not been shown to be of benefit in pediatric/ WT GIST when the group is considered as a whole (23). However, the heterogeneous nature of these tumors means that these findings need to be considered in light of the tumor biology on a case-by-case basis as the sensitivities of the numerous different mutations found in these tumors are not well studied $(26,27)$. For example, recent reports have demonstrated that SDHA-mutated GIST subtype is imatinib responsive, likely because this mutation does not cause an inactivation of the SDH-complex (28). Multiple studies have also demonstrated that second generation TKIs show improved activity against these subsets of tumors (29), thought likely due to its broadened activity against KIT, PDGFRA, PDGFRB, and VEGFR (30). Studies by Janeway and Reichardt have also shown that secondgeneration TKIs have shown slowed progression of disease with imatinib-resistant GIST in both adult and pediatric patients $(31,32)$.

There are also emerging therapies existing outside the more traditional paradigm of direct tyrosine kinase inhibition. It has been shown that about $88 \%$ cases of GIST in the pediatric population contain inactivating mutations in the genes coding for one of the four (A,B,C,D) subunits of the SDH complex (33). These GIST are referred to as SDH-deficient. This essentially causes destabilization of the SDH complex which results in increased growth signaling through IGF1R and VEGFR (34). These may prove to be beneficial target pathways for pediatric GIST and there are currently multiple therapies in various stages of clinical trials, such as ILGF1R inhibitor OSI-906 (Linsitinib) (35).

Finally, a brief comment should be made on the role of more traditional chemotherapeutic regimens and radiation therapy as they play a role in the treatment in other types of soft tissue sarcomas (36). Prior to the development of the TKI, treatment of GIST was limited to the cytotoxic chemotherapy regimens which were utilized in other forms of soft tissue sarcomas at that time. As we have become aware of the developed resistance to TKI therapy which occurs in treated adults, and of non-responsiveness to TKI in pediatric/WT GIST, several studies have again looked at treatment with cytotoxic agents when TKI therapy fails. However, their results have been consistent with prior studies and have failed to show any significant clinical improvement in this cohort of patients $(37,38)$. There are several newer cytotoxic agents in various stages of clinical trials but none has shown outstanding promise (39). Radiotherapy for GIST suffers from the drawbacks of a widely irradiated field, difficulties in targeting lesions affixed to mobile organs, and a high rate of gastrointestinal sideeffects. However, its use in the treatment of palliation for metastatic lesions is acknowledged in societal guidelines (40). Additionally, some recent studies performed on relatively large cohorts suggest that GIST may not be as resistant to radiotherapy as previously thought (41). Some reports also show that clinical situations arise where radiation may be able to play a key role as a salvage or palliation therapy 
for symptomatic, metastatic disease that is not amenable to surgery (42).

\section{Summary}

In summary, GIST are rare tumors in the pediatric population. Complete surgical resection, without pseudocapsule rupture, remains the optimal management. Surgical planning must consider long term morbidity and surgeons should pursue organ sparing methodology whenever possible. Primary resection should proceed even in cases with known metastasis as removal of primary tumor shows a clear survival benefit. Although metastatic lesions should be biopsied if discovered, radical metastasectomy should not be attempted because of the high morbidity and no clear difference in outcome. Sampling of draining lymph node basins is recommended for staging in the pediatric population. Surgical technique should be chosen based on surgeon comfort and tumor size. Although extremely beneficial in the adult population, adjuvant therapy has not yielded the same results in the pediatric population due to difference in cell biology and genetic mutations. Referral to a high-volume center is critical for these patients as some pediatric GISTs may be responsive to Imatinib, second generation TKIs, or other new and emerging adjuvant therapies.

\section{Acknowledgements}

None.

\section{Footnote}

Conflicts of Interest: The authors have no conflicts of interest to declare.

\section{References}

1. Ma GL, Murphy JD, Martinez ME, et al. Epidemiology of gastrointestinal stromal tumors in the era of histology codes: results of a population-based study. Cancer Epidemiol Biomarkers Prev 2015;24:298-302.

2. Søreide K, Sandvik OM, Søreide JA, et al. Global epidemiology of gastrointestinal stromal tumours (GIST): A systematic review of population-based cohort studies. Cancer Epidemiol 2016;40:39-46.

3. Perez EA, Livingstone AS, Franceschi D, et al. Current incidence and outcomes of gastrointestinal mesenchymal tumors including gastrointestinal stromal tumors. J Am Coll Surg 2006;202:623-9.

4. Benesch M, Wardelmann E, Ferrari A, et al. Gastrointestinal stromal tumors (GIST) in children and adolescents: A comprehensive review of the current literature. Pediatr Blood Cancer 2009;53:1171-9.

5. Kaemmer DA, Otto J, Lassay L, et al. The Gist of literature on pediatric GIST: review of clinical presentation. J Pediatr Hematol Oncol 2009;31:108-12.

6. Janeway KA, Liegl B, Harlow A, et al. Pediatric KIT wildtype and platelet-derived growth factor receptor alphawild-type gastrointestinal stromal tumors share KIT activation but not mechanisms of genetic progression with adult gastrointestinal stromal tumors. Cancer Res 2007;67:9084-8.

7. Demetri GD, Benjamin RS, Blanke CD, et al. NCCN task force report: management of patients with gastrointestinal stromal tumor-update of the NCCN clinical practice guidelines. J Natl Compr Canc Netw 2007;5 Suppl 2:S129; quiz S30.

8. Nishida T, Blay JY, Hirota S, et al. The standard diagnosis, treatment, and follow-up of gastrointestinal stromal tumors based on guidelines. Gastric Cancer 2016;19:3-14.

9. Mullassery D, Weldon CB. Pediatric/"Wildtype" gastrointestinal stromal tumors. Semin Pediatr Surg 2016;25:305-10.

10. Kim BJ, Kays JK, Koniaris LG, et al. Understanding the critical role for surgery in the management of wildtype gastrointestinal stromal tumor (GIST). Transl Gastroenterol Hepatol 2017;2:91.

11. Catena F, Di Battista M, Ansaloni L, et al. Microscopic margins of resection influence primary gastrointestinal stromal tumor survival. Onkologie 2012;35:645-8. Erratum in: Onkologie 2013;36:45.

12. Ahmed I, Welch NT, Parsons SL. Gastrointestinal stromal tumours (GIST) - 17 years experience from Mid Trent Region (United Kingdom). Eur J Surg Oncol 2008;34:445-9.

13. Gouveia AM, Pimenta AP, Capelinha AF, et al. Surgical margin status and prognosis of gastrointestinal stromal tumor. World J Surg 2008;32:2375-82.

14. Everett M, Gutman H. Surgical management of gastrointestinal stromal tumors: analysis of outcome with respect to surgical margins and technique. J Surg Oncol 2008;98:588-93. Erratum in: J Surg Oncol 2009;99:318.

15. Weldon CB, Madenci AL, Boikos SA, et al. Surgical Management of Wild-Type Gastrointestinal Stromal 
Tumors: A Report From the National Institutes of Health Pediatric and Wildtype GIST Clinic. J Clin Oncol 2017;35:523-8.

16. Agaram NP, Laquaglia MP, Ustun B, et al. Molecular characterization of pediatric gastrointestinal stromal tumors. Clin Cancer Res 2008;14:3204-15.

17. Hiki N, Yamamoto Y, Fukunaga T, et al. Laparoscopic and endoscopic cooperative surgery for gastrointestinal stromal tumor dissection. Surg Endosc 2008;22:1729-35.

18. Tsujimoto H, Yaguchi Y, Kumano I, et al. Successful gastric submucosal tumor resection using laparoscopic and endoscopic cooperative surgery. World J Surg 2012;36:327-30.

19. Fero KE, Coe TM, Fanta PT, et al. Surgical Management of Adolescents and Young Adults With Gastrointestinal Stromal Tumors: A US Population-Based Analysis. JAMA Surg 2017;152:443-51.

20. Hirota S, Isozaki K, Moriyama Y, et al. Gain-of-function mutations of c-kit in human gastrointestinal stromal tumors. Science 1998;279:577-80.

21. Perez EA, Gutierrez JC, Jin X, et al. Surgical outcomes of gastrointestinal sarcoma including gastrointestinal stromal tumors: a population-based examination. J Gastrointest Surg 2007;11:114-25.

22. Wang D, Zhang Q, Blanke CD, et al. Phase II trial of neoadjuvant/adjuvant imatinib mesylate for advanced primary and metastatic/recurrent operable gastrointestinal stromal tumors: long-term follow-up results of Radiation Therapy Oncology Group 0132. Ann Surg Oncol 2012;19:1074-80. Erratum in: Ann Surg Oncol 2012;19:2420.

23. Corless CL, Ballman KV, Antonescu CR, et al. Pathologic and molecular features correlate with longterm outcome after adjuvant therapy of resected primary GI stromal tumor: the ACOSOG Z9001 trial. J Clin Oncol 2014;32:1563-70. Erratum in: J Clin Oncol 2014;32:3462.

24. Falor A, Arrington AK, Luu C, et al., Massive IntraAbdominal Imatinib-Resistant Gastrointestinal Stromal Tumor in a 21-Year-Old Male. Case Reports in Medicine 2013;Article ID 373981:5.

25. Miettinen M, Wang ZF, Sarlomo-Rikala M, et al. Succinate dehydrogenase-deficient GISTs: a clinicopathologic, immunohistochemical, and molecular genetic study of 66 gastric GISTs with predilection to young age. Am J Surg Pathol 2011;35:1712-21.

26. Yantiss RK, Rosenberg AE, Sarran L, et al. Multiple gastrointestinal stromal tumors in type I neurofibromatosis: a pathologic and molecular study. Mod Pathol 2005;18:475-84.

27. Miranda C, Nucifora M, Molinari F, et al. KRAS and BRAF mutations predict primary resistance to imatinib in gastrointestinal stromal tumors. Clin Cancer Res 2012;18:1769-76.

28. Szucs Z, Thway K, Fisher C, et al. Molecular subtypes of gastrointestinal stromal tumors and their prognostic and therapeutic implications. Future Oncol 2017;13:93-107.

29. Agaram NP, Wong GC, Guo T, et al. Novel V600E BRAF mutations in imatinib-naive and imatinib-resistant gastrointestinal stromal tumors. Genes Chromosomes Cancer 2008;47:853-9.

30. Sakamoto KM. Su-11248 Sugen. Curr Opin Investig Drugs 2004;5:1329-39.

31. Janeway KA, Albritton KH, Van Den Abbeele AD, et al. Sunitinib treatment in pediatric patients with advanced GIST following failure of imatinib. Pediatr Blood Cancer 2009;52:767-71.

32. Reichardt $P$, Kang YK, Rutkowski $P$, et al. Clinical outcomes of patients with advanced gastrointestinal stromal tumors: safety and efficacy in a worldwide treatment-use trial of sunitinib. Cancer 2015;121:1405-13.

33. Boikos SA, Pappo AS, Killian JK, et al. Molecular Subtypes of KIT/PDGFRA Wild-Type Gastrointestinal Stromal Tumors: A Report From the National Institutes of Health Gastrointestinal Stromal Tumor Clinic. JAMA Oncol 2016;2:922-8.

34. Gill AJ. Succinate dehydrogenase (SDH) and mitochondrial driven neoplasia. Pathology 2012;44:285-92.

35. Vadakara J, von Mehren M. Gastrointestinal stromal tumors: management of metastatic disease and emerging therapies. Hematol Oncol Clin North Am 2013;27:905-20.

36. Spira AI, Ettinger DS. The use of chemotherapy in softtissue sarcomas. Oncologist 2002;7:348-59.

37. Trent JC, Beach J, Burgess MA, et al. A two-arm phase II study of temozolomide in patients with advanced gastrointestinal stromal tumors and other soft tissue sarcomas. Cancer 2003;98:2693-9.

38. Demetri GD, Reichardt P, Kang YK, et al. Efficacy and safety of regorafenib for advanced gastrointestinal stromal tumours after failure of imatinib and sunitinib (GRID): an internation;381:295-302.

39. Alturkmani HJ, Pessetto ZY, Godwin AK. Beyond standard therapy: drugs under investigation for the treatment of gastrointestinal stromal tumor. Expert Opin Investig Drugs 2015;24:1045-58.

40. Gastrointestinal stromal tumours: ESMO Clinical Practice 
Guidelines for diagnosis, treatment and follow-up. Ann Oncol 2014;25: iii21-6.

41. Cuaron JJ, Goodman KA, Lee N, et al. External beam radiation therapy for locally advanced and metastatic

doi: $10.21037 / \operatorname{tgh} .2018 .07 .09$

Cite this article as: Willobee BA, Quiroz HJ, Sussman MS, Thorson CM, Sola JE, Perez EA. Current treatment strategies in pediatric gastrointestinal stromal cell tumor. Transl Gastroenterol Hepatol 2018;3:53. gastrointestinal stromal tumors. Radiat Oncol 2013;8:274.

42. Gatto L, Nannini M, Saponara M, et al. Radiotherapy in the management of gist: state of the art and new potential scenarios. Clin Sarcoma Res 2017;7:1. 